\title{
Hormone antagonists for two-pronged attack on BPH
}

New data published in the Journal of Urology have shown a positive effect of combined therapy with antagonists of growth hormone-releasing hormone (GHRH) and luteinizing hormonereleasing hormone $(\mathrm{LHRH})$ on rat models of $\mathrm{BPH}$.

Following on from previous research that identified potential roles for GHRH and LHRH in BPH development, Rick and colleagues evaluated the in vivo effects of the GHRH antagonist JMR-132, the LHRH antagonist cetrorelix and combined JMR-132 and cetrorelix therapy on testosterone-induced $\mathrm{BPH}$ in adult male Wistar rats.

Treatment with both antagonists resulted in moderate prostate shrinkage $(\sim 20 \%)$ and improvements in associated biochemistry. Combination treatment had a cumulative effect, resulting in a $30 \%$ shrinkage in prostate volume $(P<0.01)$. The size of epithelial areas in the ventral prostate, prostatic DNA content and proliferating cell nuclear antigen (PCNA) levels were also reduced. Furthermore, levels of prostatic and serum PSA and six transmembrane epithelial antigen of the prostate (STEAP) - a cell surface antigen that has been linked to prostate

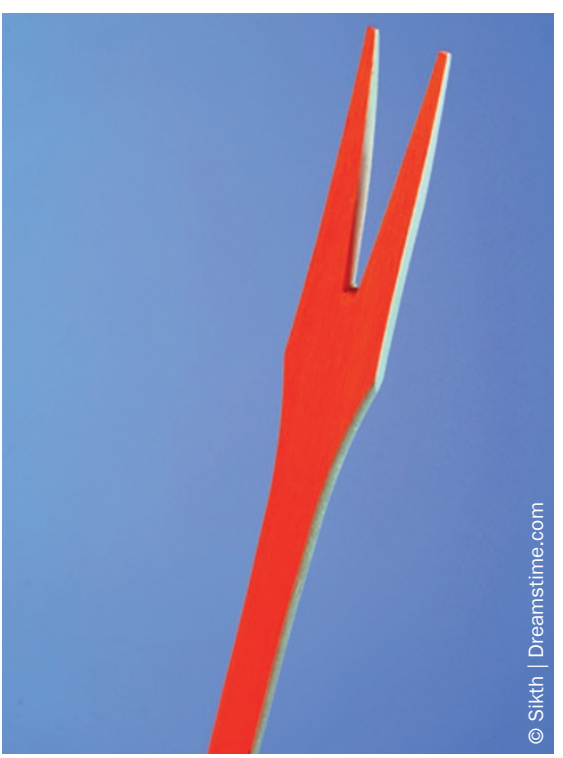

cancer-decreased substantially in response to combination treatment, and proapoptotic and antiproliferative effects were also observed using this treatment approach, with significant transcriptional downregulation of antiapoptotic Bcl-2 and upregulation of proapoptotic Bax.

The suboptimal efficacy of current standard-of-care treatments for BPH-including $\alpha$-adrenergic blockers and $5 \alpha$-reductase inhibitors such as finasteride-means that novel therapeutic approaches are actively being sought for this condition. These preliminary data support the continued development of combined GHRH antagonist and LHRH antagonist therapy for $\mathrm{BPH}$.

Melanie Clyne

Original article Rick, F. G. et al. Combining growth hormone-releasing hormone antagonist with luteinizing hormone-releasing hormone antagonist greatly augments benign prostatic hyperplasia shrinkage. J. Urol. 187, 1498-1504 (2012) 